\title{
Selective citation practices in imaging research: Are diagnostic accuracy studies with positive titles and conclusions cited more often?
}

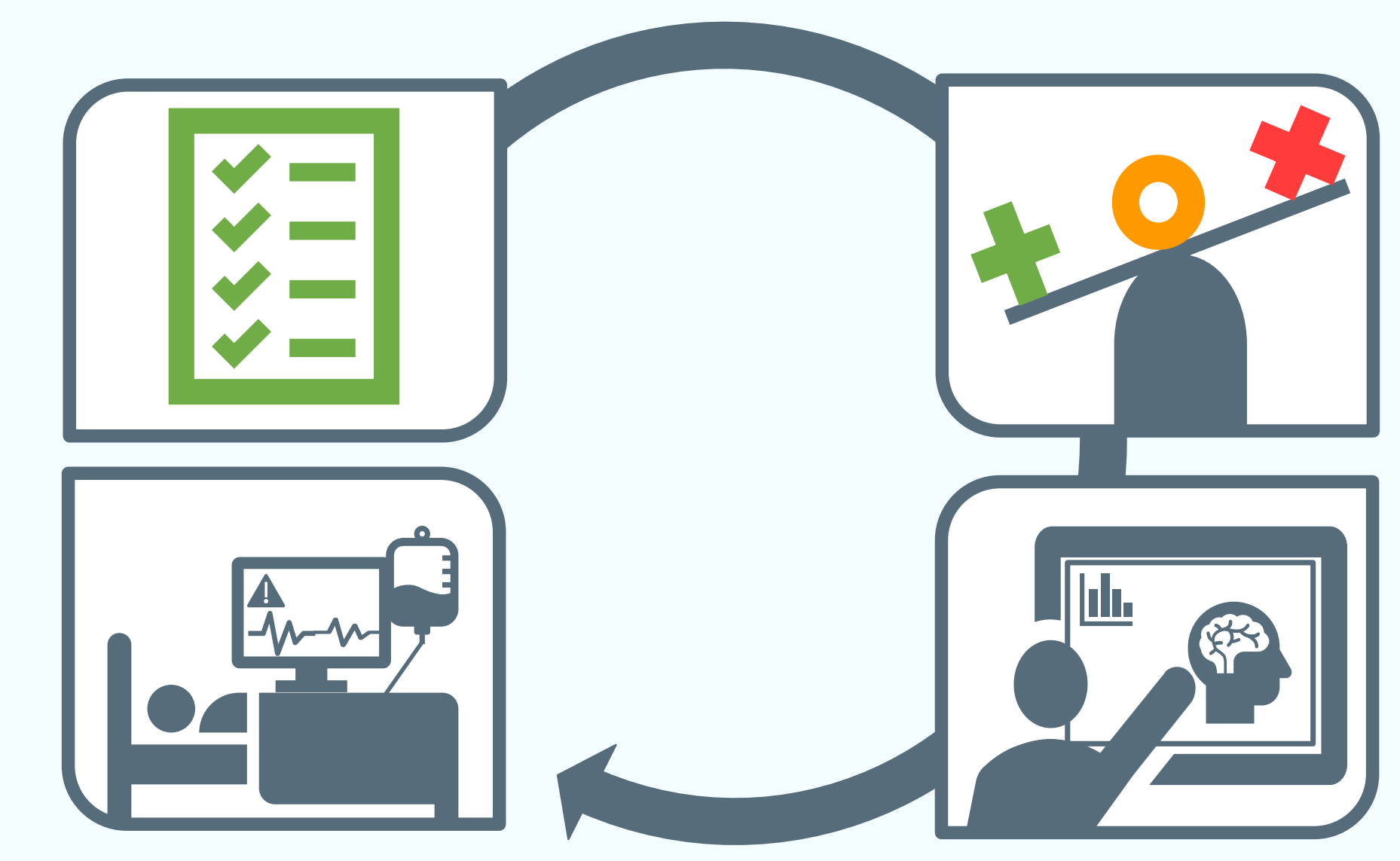

Lee Treanor $^{1,2}$, Robert Frank ${ }^{1,2}$, Jean-Paul Salameh ${ }^{3,4}$, Anahita Dehmoobad $^{1,2}$, Trevor McGrath $^{1,2}$, Noémie Kraaijpoel ${ }^{5}$, Patrick Bossuyt ${ }^{5}$, and Matthew McInnes ${ }^{1,3}$

Division of Radiology, The Ottawa Hospital; 2. Faculty of Medicine, University of Ottawa; 3. Ottawa Hospital Research Institute; 4. School of Epidemiology and Public Health, University of Ottawa; 5. Amsterdam Public Health Research Institute, University of Amsterdam, Amsterdam, Netherlands

Accepted for publication to AJR in February 2019

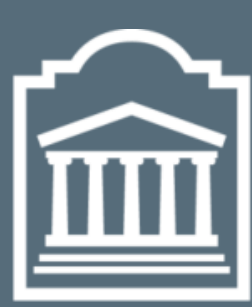

uOttawa
Lee Treanor, MS2 ltrea080@uottawa.ca Supervisor: Dr. Matthew McInnes 


\section{Disclosure of Conflict of Interest}

I do not have a relationship with a for-profit and/or a not-for-profit organization to disclose

Lee Treanor, MS2

ltrea080@uottawa.ca 


\section{Objective}

To assess whether primary diagnostic accuracy studies with positive conclusions or titles are cited more frequently than those with negative (or neutral) conclusions or titles in the imaging diagnostic accuracy literature. 


\section{Introduction}

- Studies may be cited more often, based on their results. These selective citation practices have been well-documented in biomedical sciences and among trials of therapeutic interventions, ${ }^{1,2}$ but there has been little work towards identifying its presence in the imaging diagnostic accuracy literature.

- In order to be thoroughly informed on a topic, clinicians and radiologists require a balanced exposure of all relevant evidence. Well-conducted diagnostic accuracy systematic reviews consider all relevant articles on a topic. In contrast, other studies may utilize a potentially biased approach to study inclusion or citation (see Figure 1).

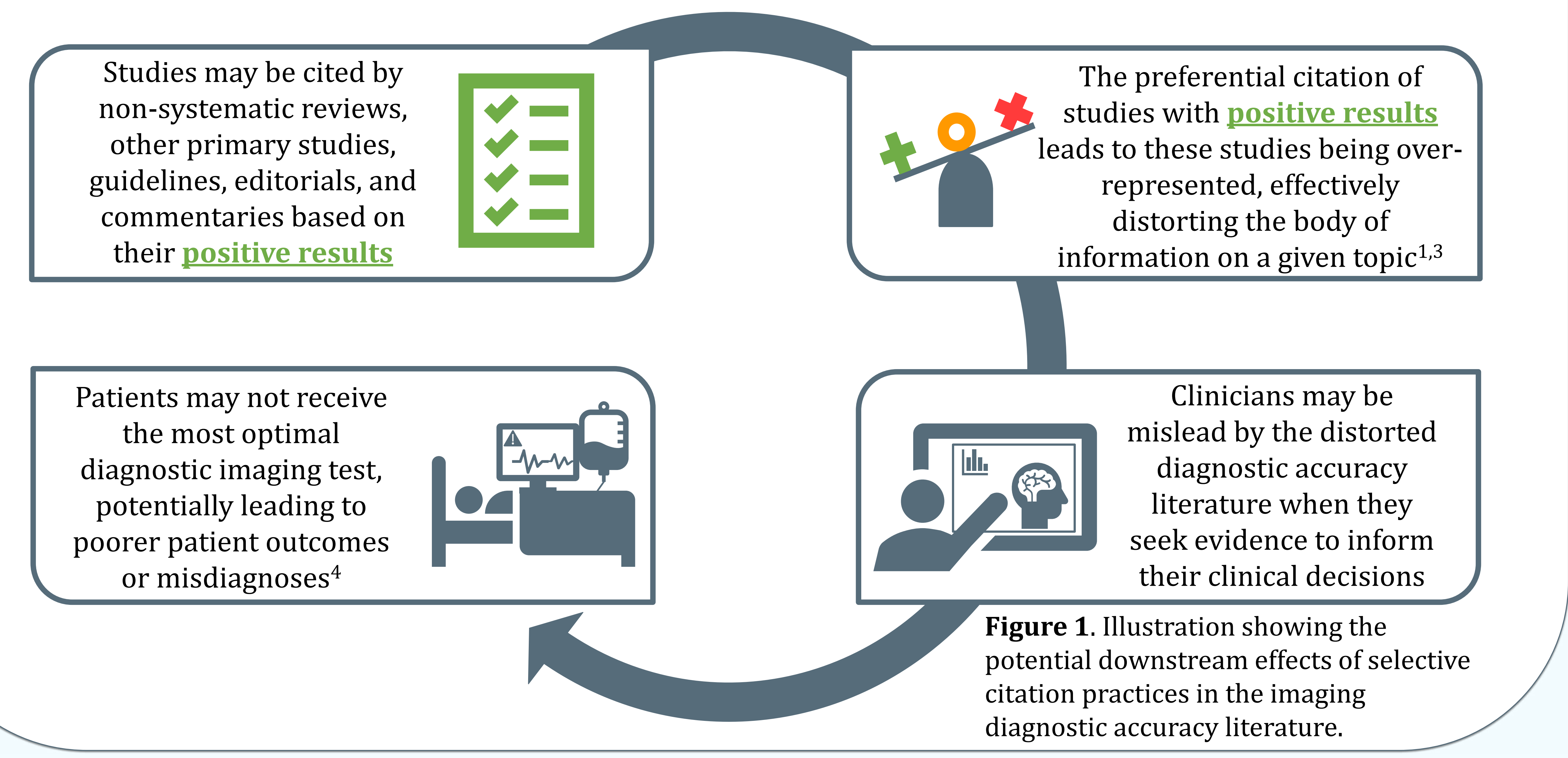




\section{Methods}

- Medline was searched for meta-analyses of diagnostic accuracy studies published in imaging journals from Jan-2005Apr-2016. Primary studies (assessing the diagnostic accuracy of at least one imaging test) from the meta-analyses were screened.

- Conclusions/titles were classified according to a published classification scheme ${ }^{5}$

- Each abstract was classified independently by two authors.

- The components of the conclusion that were classified were the summary statement (a statement about accuracy, eg. "test is accurate") and implication for practice (a statement not directly reflected in the results, eg. " test should be used"). Examples are seen in Table 1.

Table 1. Examples of conclusion classification.

\begin{tabular}{|c|c|c|}
\hline $\begin{array}{l}\text { Conclusion } \\
\text { positivity }\end{array}$ & Summary statement (accuracy) & Implication for practice statement \\
\hline Positive & $\begin{array}{l}\text { - SPECT is accurate for the detection and } \\
\text { localization of coronary artery disease. }\end{array}$ & $\begin{array}{l}\text { - SPECT should be the first-line imaging modality in } \\
\text { assessing coronary artery disease. }\end{array}$ \\
\hline $\begin{array}{l}\text { Positive with } \\
\text { qualifier }\end{array}$ & $\begin{array}{l}\text { - MRI can accurately detect colorectal masses, } \\
\text { but only if they are above } 10 \mathrm{~mm} \text { in diameter. }\end{array}$ & $\begin{array}{l}\text { - MR colonography appears to be very useful. However, } \\
\text { further research should be performed. }\end{array}$ \\
\hline Neutral & $\begin{array}{l}\text { - Breast MRI has good sensitivity but } \\
\text { mediocre specificity in detecting breast } \\
\text { cancer. }\end{array}$ & $\begin{array}{l}\text { - Breast MRI is moderately useful and may help in } \\
\text { some patients. }\end{array}$ \\
\hline Negative & $\begin{array}{l}\text { - Integrated PET-CT is inaccurate in } \\
\text { predicting hilar lymph node status. }\end{array}$ & $\begin{array}{l}\text { - Integrated PET-CT should not be used in the } \\
\text { evaluation of hilar lymph nodes. }\end{array}$ \\
\hline
\end{tabular}




\section{Results (descriptive statistics)}

- 995 primary studies from 94 meta-analyses remained after applying inclusion and exclusion criteria. Summary characteristics of the included studies are shown in Table 3, with graphs of conclusion and title positivity in Figure 2.

Table 3. Summary characteristics of the 995 included studies.

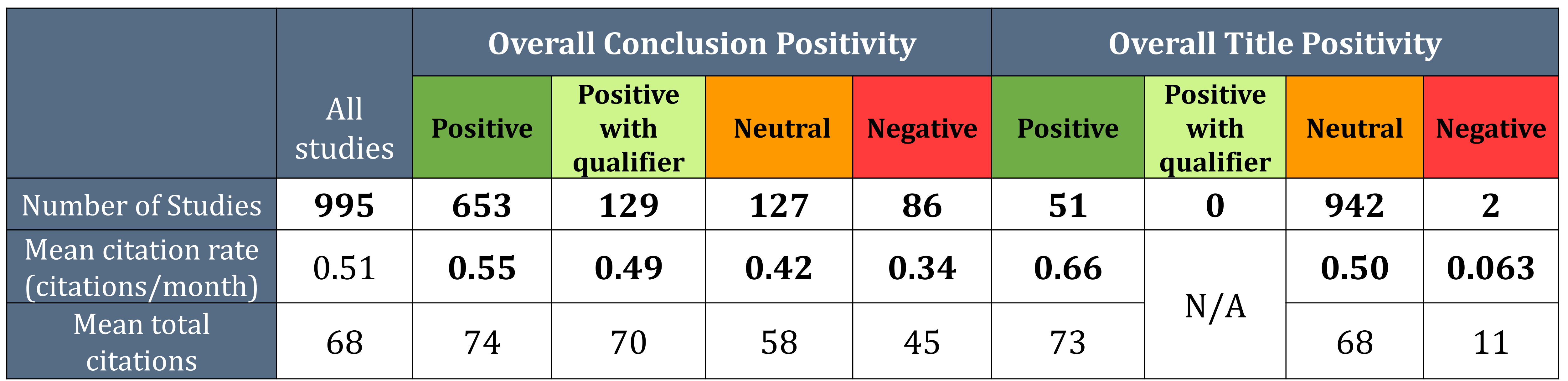

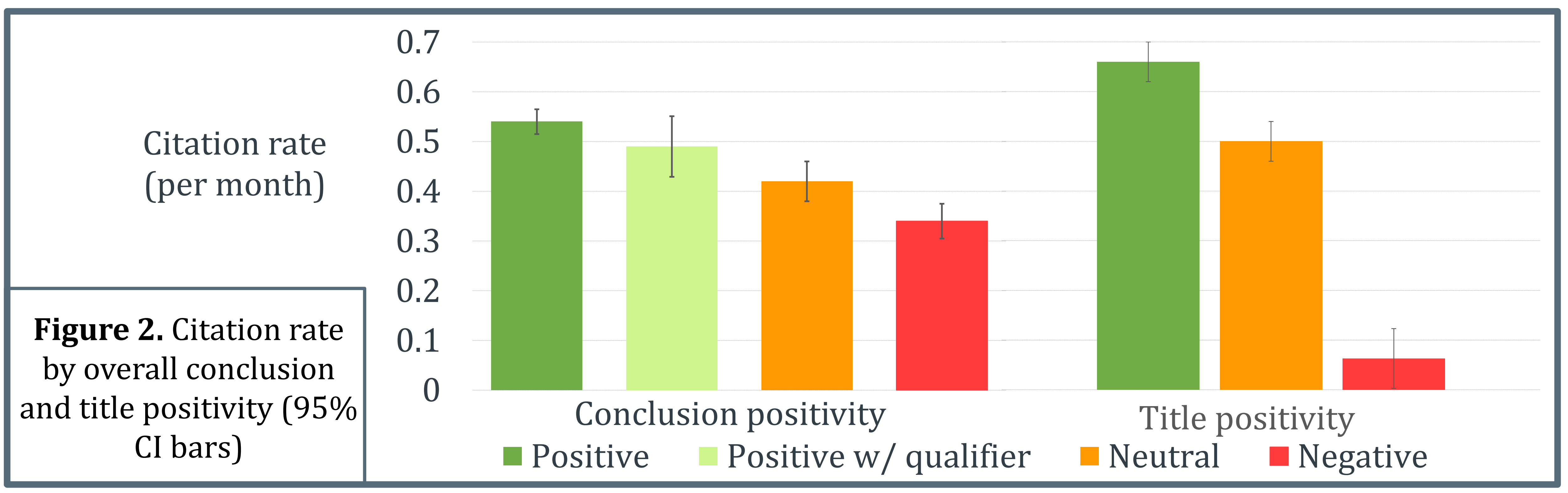




\section{Results (regression analysis)}

- A negative binomial regression analysis (controlling for confounding variables) was performed to obtain regression coefficients for the association between title/conclusion positivity and citation rate, as seen in Table 4; p-values were obtained via likelihood ratio testing.

- The eight following confounding variables were controlled for, as they are known to be potentially associated with citation rate based on prior research:

- imaging modality

- organ-based subspecialty

- 2016 journal impact factor

- study design (prospective, retrospective, or not reported),
- sample size

- source meta-analysis

- publication order

- Youden's index

Table 4. Regression analysis and likelihood ratio tests results.

\begin{tabular}{|c|c|c|c|}
\hline Variable & Regression Coefficient & Standard Error & $p$ Value \\
\hline & \multicolumn{3}{|c|}{ Outcomes of Interest } \\
\hline \multicolumn{4}{|c|}{ Overall Conclusion Positivity } \\
\hline Positive & 0.19 & 0.079 & \multirow{3}{*}{$0.0312^{*}$} \\
\hline Neutral & 0.078 & 0.096 & \\
\hline Negative & \multicolumn{2}{|c|}{ Reference category } & \\
\hline \multicolumn{4}{|c|}{ Overall Title Positivity } \\
\hline Positive & 1.1 & 0.57 & \multirow{3}{*}{$0.0306^{*}$} \\
\hline Neutral & 0.91 & 0.56 & \\
\hline Negative & \multicolumn{2}{|c|}{ Reference category } & \\
\hline
\end{tabular}




\section{Discussion and conclusion}

- Studies with positive conclusions and titles were found to be cited preferentially in the imaging literature. Even when controlling for potential confounders, citation rate was significantly associated with conclusion and title positivity.

- The preferential citation of positive results may distort the diagnostic accuracy literature, leading to an unwarranted increase in the perceived performance of a given diagnostic test. Ultimately, this may adversely affect patient outcomes or even lead to misdiagnosis.

- Of course, the clinical impact of the additional citations would vary depending on the source of citation; the impact being potentially greatest if additional citations of positive results are in clinical practice guidelines or 'expert opinion' reviews by respected authors.

- There were a high proportion of studies with positive conclusions found (79\%).

- The high proportion of positive conclusions may be particularly prevalent in diagnostic accuracy research as there is typically no threshold for statistical significance (e.g. p<0.05), unless there is a well-defined comparator test; instead, there is often an interpretation of diagnostic accuracy. This high proportion of positive studies may also be in part due to publication bias (topic of further research).

- Clinicians, researchers, and journal editors should be aware of the presence of and potential impact of selective citation practices. Promoting high quality systematic reviews as optimal sources for guidelines and expert opinion pieces may mitigate this potentially harmful phenomenon.
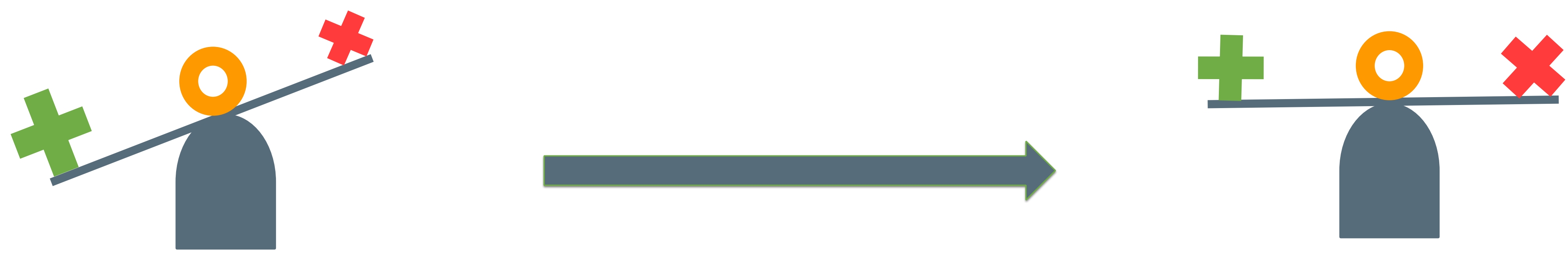


\section{Limitations}

- A potential limitation of the study was the subjective nature of classification. We tried to limit this subjectivity by using a previously established scheme, piloting prior to extraction, and by conducting independent extractions in duplicate. Our analysis was limited to studies that were published, so we were unable to account for publication bias as a potential confounding variable.

\section{References}

1. Duyx B, Urlings MJE, Swaen GMH, Bouter LM, Zeegers MP. Scientific citations favor positive results: a systematic review and meta-analysis. J Clin Epidemiol. 2017;88:92-101. Epub 2017/06/08. doi: 10.1016/j.jclinepi.2017.06.002. PubMed PMID: 28603008.

2. Misemer BS, Platts-Mills TF, Jones CW. Citation bias favoring positive clinical trials of thrombolytics for acute ischemic stroke: a crosssectional analysis. Trials. 2016;17(1):473. Epub 2016/09/28. doi: 10.1186/s13063-016-1595-7. PubMed PMID: 27677444; PubMed Central PMCID: PMCPMC5039798.

3. Greenberg SA. How citation distortions create unfounded authority: analysis of a citation network. BMJ. 2009;339:b2680. Epub 2009/07/20. PubMed PMID: 19622839; PubMed Central PMCID: PMCPMC2714656.

4. Steneck NH. Fostering integrity in research: definitions, current knowledge, and future directions. Sci Eng Ethics. 2006;12(1):53-74. PubMed PMID: 16501647.

5. McGrath TA, McInnes MDF, van Es N, Leeflang MMG, Korevaar DA, Bossuyt PMM. Overinterpretation of Research Findings: Evidence of "Spin" in Systematic Reviews of Diagnostic Accuracy Studies. Clin Chem. 2017;63(8):1353-62. Epub 2017/06/12. doi: 10.1373/clinchem.2017.271544. PubMed PMID: 28606911.

6. Dilauro M, McInnes MD, Korevaar DA, van der Pol CB, Walther S, Quon J, Kurowecki D, Bossuyt PM. Is There an Association between STARD Statement Adherence and Citation Rate? Radiology. 2016;280(1): 62-67. Epub 2016/02/02. doi: 10.1148/radiol.2016151384. PubMed PMID: 26836050.

\section{Special thanks to Dr. McInnes and the team members}

